\title{
Implementation of Smart Agriculture using CloudIoT and its Geotagging on Android Platform
}

\author{
Palle Divya Vani ${ }^{a}$, Kanchi Raghavendra Rao ${ }^{\text {b* }}$ \\ ${ }^{a}$ Sri Krishnadevaraya University, Anantapur,515003, India \\ ${ }^{b}$ Sri Krishnadevaraya University, Anantapur, 515003, India
}

Received: 08 August 2017; Accepted: 08 January 2018; Published: 08 March 2019

\begin{abstract}
Agriculture is the backbone of several countries like India and most of the economy depends directly or indirectly on agriculture for a living. But these days several crops are significantly low and farmers are difficult to analyze the variations of soil moisture on yield in every day. The solution of the mentioned problem is to place the sensors in the yield and to collect the readings of moisture in every day by implementing the technologies: Internet of Things (IoT), Cloud Computing technology and GSM (Global Service Message) communication. In this paper, explain how these technologies and GSM will help the farmers in areas where there is an acute water problem or where water conservation is essential. The system is used Wi-Fi and GSMbased communications to send the sensor data to Cloud and mobile phones. The hardware is developed using TI's First Wi-Fi on-chip microcontroller: CC3200 LaunchPad, SIM900A GSM module, soil moisture sensor, motor and mobile phone. The software is developed using Energia IDE. Thing Speak Cloud Computing technology is used in this work for storing the sensor values in. CSV format and also shows the graphical representation of the real-time variation of moisture on mobile using mobile application and Laptop, SIM 900A module is used sent the moisture levels to the farmer's registered mobile numbers. The system also automatically turns ON/OFF the motor based on the variation of moisture on yields. The whole system is easily implemented on yields and gets the moisture information even in remote areas in the world.
\end{abstract}

Index Terms: CC3200 LaunchPad, SIM900A GSM Module, Internet of Things (IoT), Cloud Computing Technology, FC-28 Soil Moisture Sensor.

(C) 2019 Published by MECS Publisher. Selection and/or peer review under responsibility of the Research Association of Modern Education and Computer Science.

\footnotetext{
* Corresponding author.

E-mail address: vani.divya63@gmail.com, kanchiraghavendrarao@gmail.com
} 


\section{Introduction}

In ancient days, the crop production was done by farmers which require lots of hard work and attention and they obtain less productivity, more consuming of water for production and time consuming. By the development of technologies such as the Internet of Things (IoT), Cloud Computing technology, Wireless sensor networks etc are implemented in agriculture production. This will reduce the human efforts, minimal use of water and better production [1]. IoT is a new technology and is becoming an increasingly growing topic of conversation both in workspace and outside of it [2]. IoT technology which brings objects into the internet, and exchanges information anytime and anyplace [3]. IoT has three basic characteristics and four elements such as information collection, two-way transmission, treatment the information and feedback. The characteristics are: First, miniaturization, with the technology available in hands of users through smart devices anywhere, anytime. Secondly, interconnectivity, and thirdly, intelligence in the applications and services that make use of the large amount data created via the sensor networks and converts this data into useful information to enable real-time decision making and scientific discoveries. IoT is widely used in intelligent transportation, public safety, personal health, home automation systems, environment protection, agriculture systems and other fields [4]. Cloud computing can be most promising and cost-effective solution to connect, manage and track the IoT. Cloud computing technology has three basic models such as IaaS, PaaS, SaaS and these are suitable for IoT applications [5]. Using the Cloud Computing vast amount of sensor data can be processed, analyzed, and stored using computational and storage resources of the Cloud. Cloud has three dimensions-volume, variety and velocity. Volume refers to large transactions, variety refers to data types (tabular data, documents, video, images, emails, stock ticker data), originating from social media and mobile, and velocity refers to the structuring of data and making it available for access and delivery [6]. The cloud computing is used for processing and analyzing the data. The data on Cloud is displayed on the website in time series graphs; each graph represents the corresponding sensing input information. The website performs the raw sample data to units for displaying in the graphs. In UDP (User Datagram Protocol) packets produced at the gateway encapsulating sample data to be sent to windows based server [7]. An application running on the server uses the standard socket interface to receive UDP packets on an arbitrary port, and stores the relevant information in the MySQL database. The database has four columns: Source address, time, and source channel and sample data. Rows are added to this table for each UDP packet received. This allows samples to be stored by time, sensor node and sensor channel. A wireless sensor network (WSN) is the lowest layer IoT architecture. The practical application of WSN is mostly based on Bluetooth, Wi-Fi and Zigbee. In this paper, the hardware is developed using CC3200 LaunchPad, sensors, GSM module, IoT, Cloud computing technologies are used and the moisture information is viewed in real time on PC and at the same time the motor is controlled based on the moisture values, the variation of moisture values is sent to the farmers mobile phones. The motivation behind such a project is mainly to reduce the wastage water and it is helpful for farmers, by knowing the field situation in real-time through SMS (Short message service) in offline mode using SIM900A GSM Module and Wi-Fi technology that allows the sensor data is sent cloud computing technology. In Cloud computing technology the moisture values are seen in android mobile in online. Therefore, the developed system is helpful for the formers in both online as well as SMS.

The other sections of the paper are organized as follows: section -II discussion about literature review, section-III describe the Experimental setup and section-IV explains the results and discussions and finally, conclusions are explained in section-V.

\section{Literature Review}

Several papers have appeared in the literature which discusses measurement and monitoring the agricultural parameters by using different controllers and sensors. The work carried out by some researchers related to soil moisture measurement is as follows: 
Anthony Faustine [5] et al. developed a prototype model for water quality and controlling system using wireless sensor networks. The system detects water temperature, $\mathrm{PH}$, dissolved oxygen and electrical conductivity in real-time. The sensory data send to the web-based portal and mobile through GSM module. In [6] developed GSM based smart home and digital notice board. Home appliances are controlled using a mobile phone and send the information using GSM. The digital notice board has displayed the information in an institution without any use of paper. In [7] the system is developed for agriculture monitoring and crop growth using GSM and Zigbee technology. The sensor values of the developed are sent to the Irrigation control centre (ICC) through Zigbee and crop growth is monitoring using IR transceiver. The sensor data is also sent to the mobile through GSM. In [8] an automated water irrigation control system is used to reduce the wastage of water in crop fields. The water level reaches the threshold value; the GSM module sends SMS to the farmer. In [9] an IoT based smart irrigation system is developed using an ATMEGA328P controller. The system automatically monitors and maintains the desired soil moisture on the field and also automatically pumps the water to the fields. In [9] IoT based system is developed for patient checking and reduce the need for medical staff. The system monitors the patient body temperature, blood pressure level and pulse. In [10] developed a cloud computing system for Open Educational Resources (OER). The system will have a capability for laboratory activities, syllabi, home work and assignment, assessments, lecture notes, audio visual lectures, simulation, lesson plan and text books etc.

From the above literature, the newness of the present paper is to monitor the soil moisture parameter using Wi-Fi and GSM module with Geotagging.

\section{Experimental Setup}

The total experimental setup is explained as follows:

\subsection{Block diagram}

The system is developed by using the diagram as shown in Fig.1. The system consists of CC3200 LaunchPad, FC-28 Soil moisture sensor, SIM900A GSM Module, DC motor.

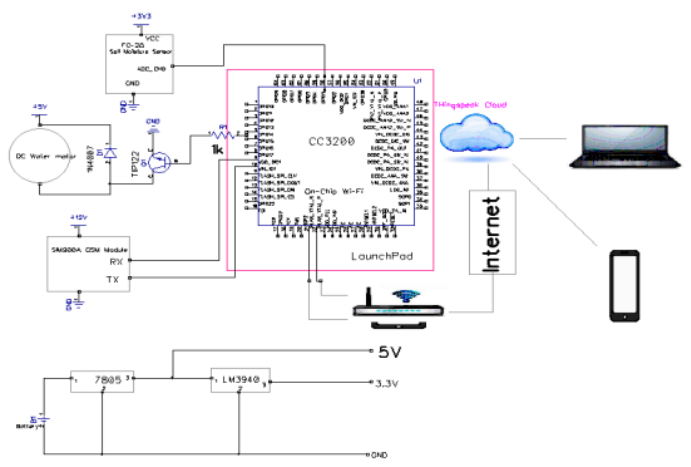

Fig.1 Block Diagram of the Developed System

From the figure, the sensor values are processed by using CC3200 LaunchPad. The sensor values are sent in a message using a mobile phone from any remote location in the world and the message is transmitted through GSM network, the SIM 900A GSM module is interfaced with CC3200. The CC3200 has on-chip Wi-Fi module, it is connected to the Wi-Fi router. By Using IP (Internet Protocol) address the sensor data is sent to the cloud computing technology, and the same data is retrieved and viewed anywhere in the world on PC and Mobile by using mobile application. The DC motor is connected to the microcontroller it is automatically ON/OFF based 
on the moisture level sensed by the sensor in agriculture Fields.

\subsection{Component Description:}

The components are used in the present work is explained below.

\subsubsection{CC3200 LaunchPad:}

Texas Instrument's CC3200 LaunchPad is the first internet on-chip Wi-Fi microcontroller. This flexible wireless chip makes it too fast and easy to build the products that connect anything to anything via the internet using Wi-Fi. The key application of CC3200 is Internet of Things (IoT) and Low power Wi-Fi. CC3200 incorporates $802.11 \mathrm{~b} / \mathrm{g} / \mathrm{n}$ radio, Baseband, Medium Access Control (MAC), Wi-Fi driver and supplicant. The chip is also contained network processor with an on-chip web server and TCP/IP stack. The device is housed in 64 pin QFN package. CC3200 integrates an 80 MHZ ARM Cortex M4 MCU. The interfacing peripherals are $I^{2}$ C, SPI, UART, GPIOs, PWM, Real-time Clock (RTC) and 4-channel 12-bit ADC. It is designed to operate two AA cells with the voltage range from 2.1-3.6V. Security is another benefit of CC3200. Wi-Fi security includes WPA2 personal, WPA2 Enterprise and WPS2. Internet security includes on-chip SSL 3.0, TLS 1.2, and X.509 [11].

\subsubsection{FC-28 Soil Moisture Sensor:}

FC-28 [12] soil moisture sensor is a simple sensor can be used to detect soil moisture present in the plant watered device. The output of FC-28 is in terms of analog voltage for measuring soil moisture. The sensor module has an LM393 driver. It is designed specially from a single supply over a wide range of voltages. The sensor module has a potentiometer to adjust the level of sensitivity. The sensor operating voltage is $3.3-5 \mathrm{~V}$.

\subsubsection{SIM900A GSM Module:}

SIM900A [13] Modem is built with Dual Band GSM/GPRS based SIM900A modem from SIMCOM. It works on frequencies 900/ $1800 \mathrm{MHz}$. SIM900A can search these two bands automatically. The frequency bands can also be set by AT Commands. The baud rate is configurable from 1200-115200 through AT command. The baud rate is used in this work is 9600 bps. The module has SIM card holder, RS232 serial control (TX, RX), Network LED, Power LED and antenna for sending/receiving signals to the SIM. It supports features like SMS, GPRS, Voice at both 900 and $1800 \mathrm{MHz}$ and the module operates 12V DC and 1-2mA.

\subsubsection{Thing Speak Cloud:}

ThingSpeak is an open data platform and API for IoT. ThingSpeak accessing the real-time data from the sensors and the data is visualized in the form of charts and graphs. The applications of this cloud are to analyze and visualize the sensor data using MATLAB, and it creates plugins, Thing Tweet, Tweet control, React, Talk Back and Thing HTTP [14]. The provision of the cloud is the data is viewed on Android or ioS mobile using the Thing View mobile application. The accessing of the cloud is explained following steps.

* The first step is to Sign up the ThingSpeak account at https://thingspeak.com

* Log in to the account and create a New Channel. The cloud automatically generates a channel Id and it reads the data by using this channel Id. Every channel has a unique channel Id and each channel provides eight data fields for displaying the data. In channel settings, select the device as either public or private and also give the specific channel name. The screenshot of channel setting window as shown in Fig 2. 


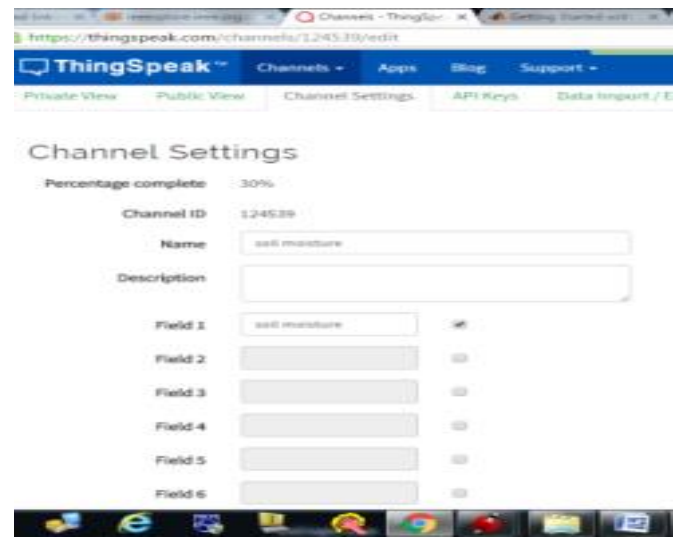

Fig.2. Screenshot of Creating a Channel Window

* After creating the channel, the cloud generates Write and Read API keys for accessing the data which is shown in Fig.3 as a screenshot.

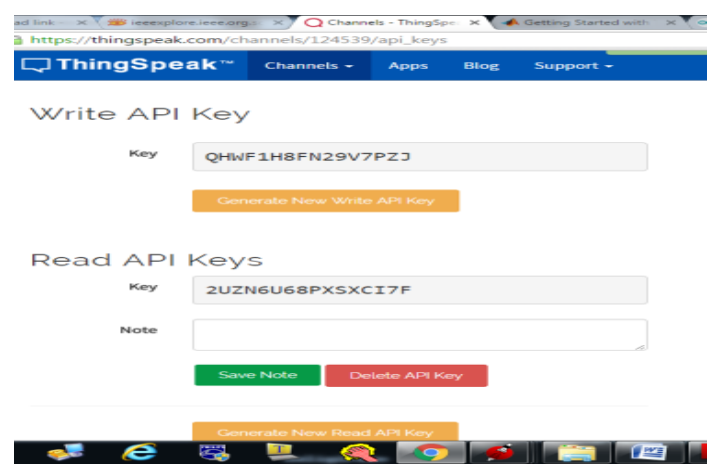

Fig.3. Screenshot of Write and Read API keys Window.

\subsection{System Architecture:}

Total Architecture of the present developed is as shown in Fig. 4.

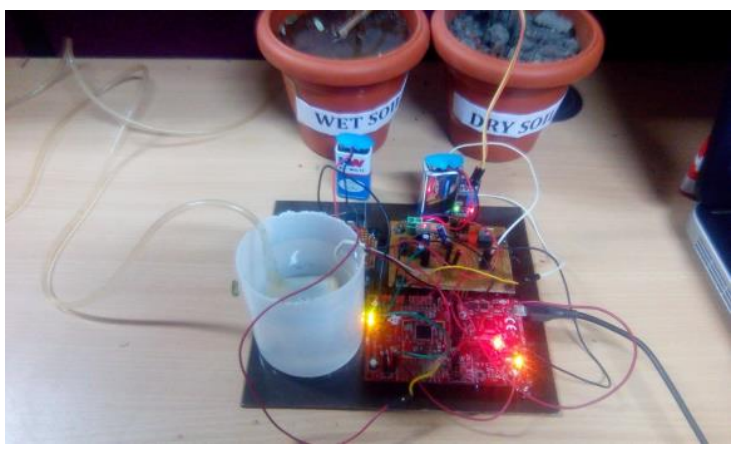

Fig.4. Total Architecture of the Present Developed System. 

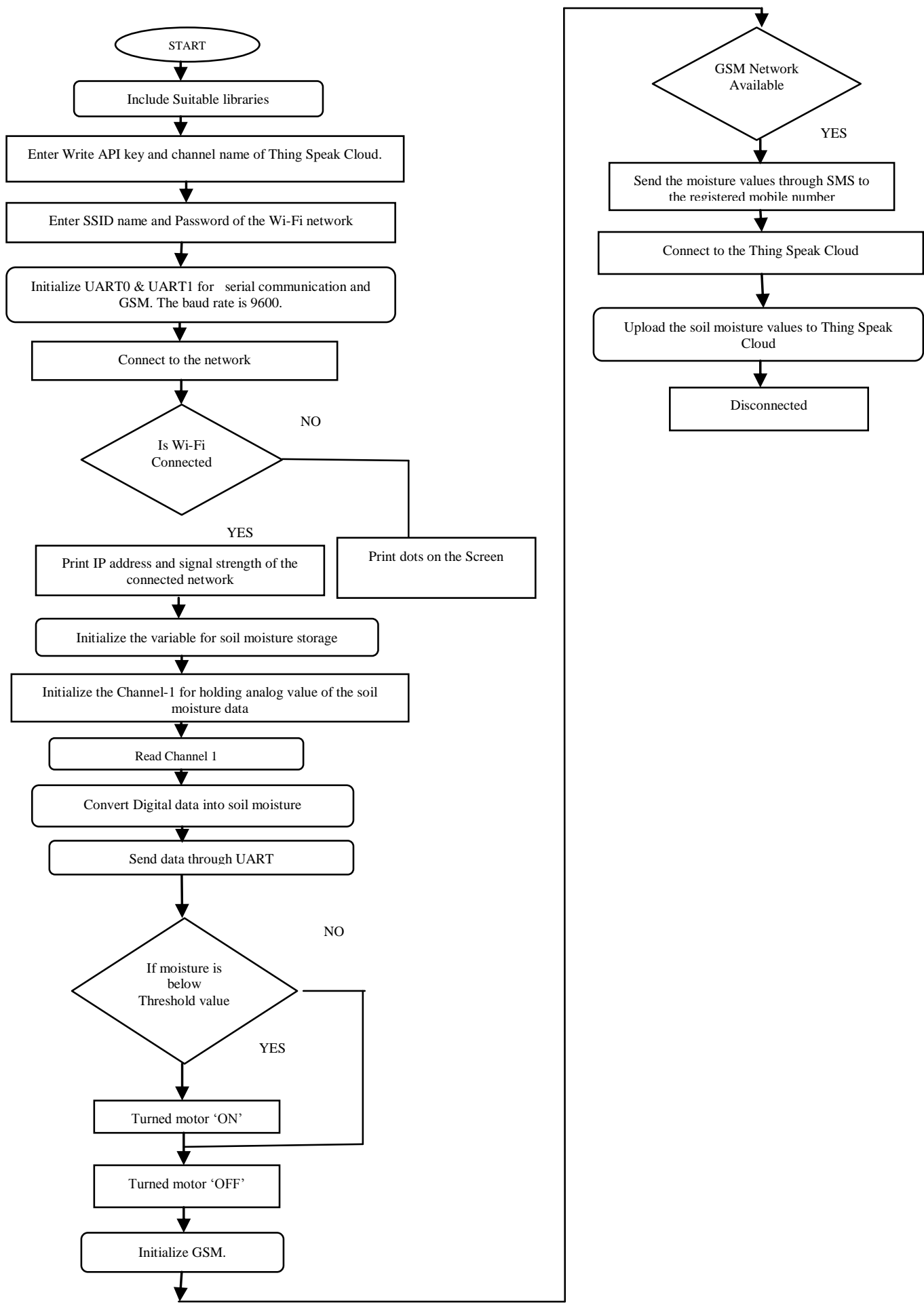

Fig.5. Flowchart of Acquiring Data from the Sensor and Data Uploaded to the cloud and processing the Data through GSM 


\subsection{Software Design:}

A software program is developed and uploaded to the microcontroller to allow the sensor values. In the present work, software is developed using Energia IDE. The process of acquiring data from the sensors and uploaded to the cloud, sent to the moisture values through SMS is shown in the flowchart as in Fig.5.

The communication between Microcontroller and GSM module is done through a serial port send AT commands [15]. The GSM module receives the commands and sends the message to the terminal through a serial port. The AT commands which are used in the present work is shown in Table 1.

Table 1. AT Commands of SIM 900A GSM Module

\begin{tabular}{|l|l|}
\hline Instructions & Meaning \\
\hline AT & Attention \\
\hline AT+CMGF & Set Module in message mode \\
\hline AT+CMGS & Send the message \\
\hline
\end{tabular}

\section{Results and Discussions}

The system was tested in Dry Soil and Wet Soil condition in a plant; it is worked perfectly in the lab. The sensor is placed at the roots of the plants. Basing on the moisture level sensed by the sensor, the motor is turned on/off. If the moisture level is below than required level, the motor is turned on and supplying the water to the plant till the soil around it attains the required moisture level and then the motor will automatically turn off. The photograph of the developed system is shown in Fig.6. The sensor data is sent to the ThingSpeak Cloud and the data is viewed in a graphical representation on the web-based portal of ThingSpeak cloud and also in mobile application.
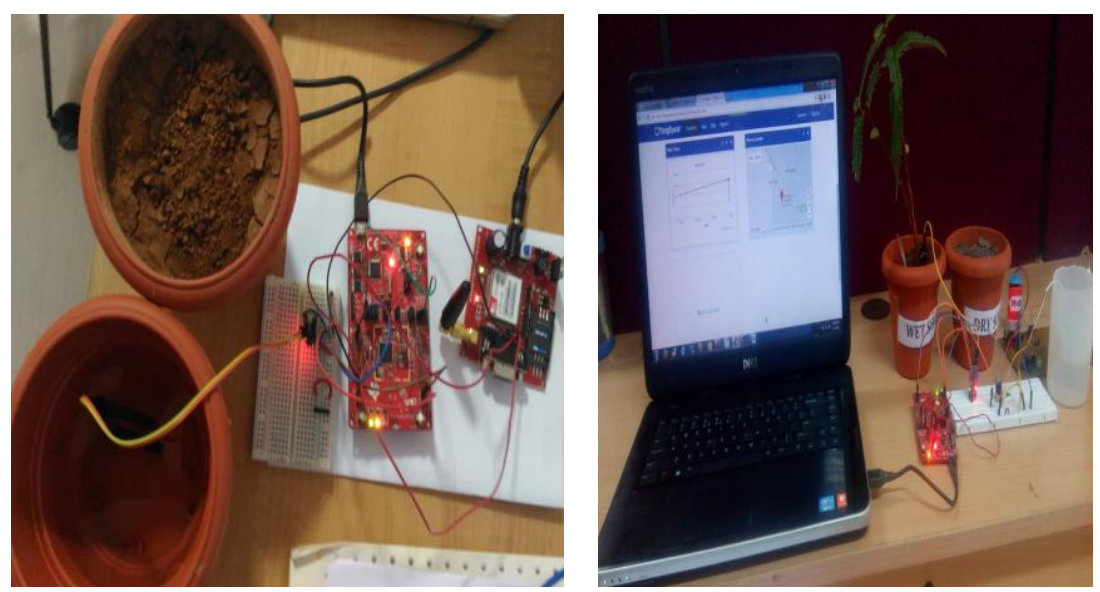

Fig.6. Photograph of the Present Developed System Tested in the lab (Left). Cloud Data Displayed on Laptop (Right)

The variation of moisture data is displayed on cloud and mobile application, and the data is also sent to a mobile phone through SMS. The Screenshots of the variation of moisture data on mobile using GSM module, the variation of moisture data on mobile is shown in Fig.7 (a) and 7(b). 


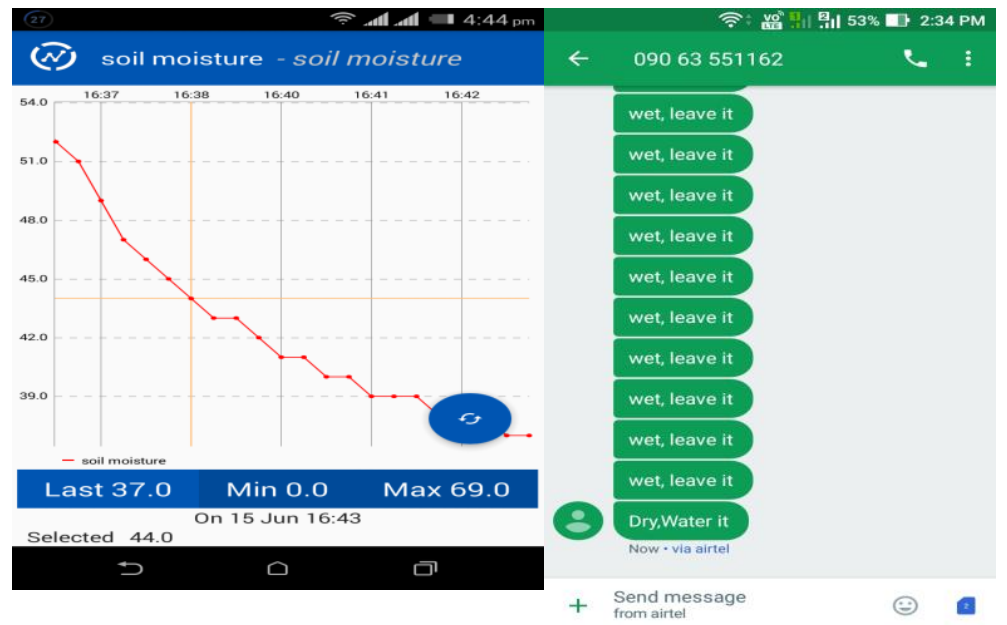

Fig.7. (a). The Variation of Moisture Data is Displayed on Cloud, Mobile by using the Mobile Application (right), (b) Screenshots of the Variation of Moisture data on Mobile through SIM900A GSM Module SIM (left) (b).

The Thing Speak Cloud has stored a large amount of data. The sensor data is stored in the cloud and retrieved the sensor data in terms of JSON or .CSV or XML file. The data on .CSV file is as shown in Fig.8.

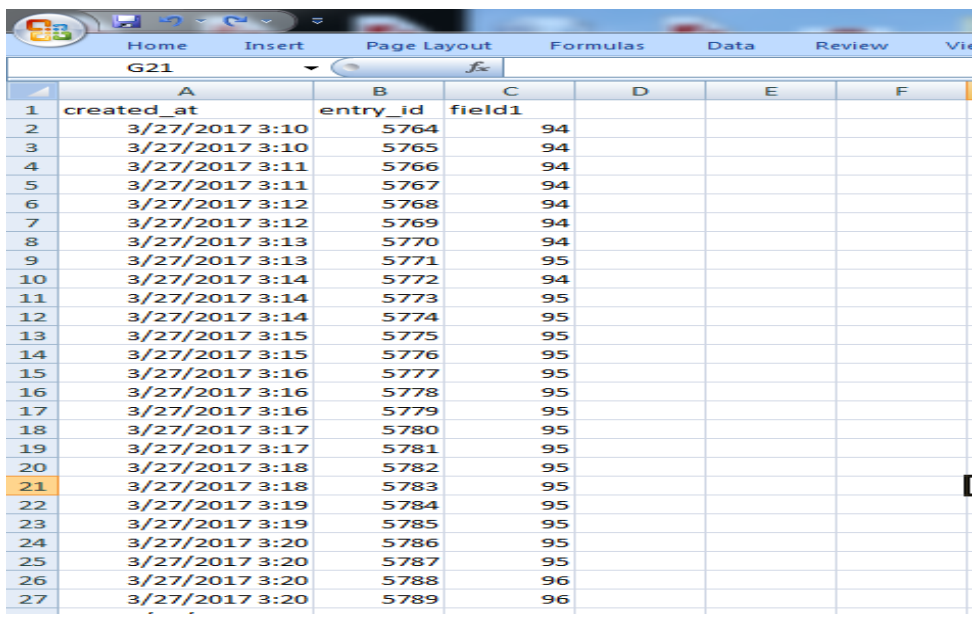

Fig.8. Retrieving of Moisture Data on Cloud

The other advantage of this paper is to Geotagging of sensors. By using Geotagging the location is easily identified by using the coordinates of the location and also it shows the map of the location on the Cloud. The ThingSpeak Cloud has interlinked with MATLAB. The MATLAB mobile application is identified by the coordinates of the location and sent these coordinates to ThingSpeak channel. The location is shown on the right of the ThingSpeak Channel. In this Paper, the total experiment is performed at SRI KRISHNADEVARAYA UNIVERSITY. The Geotagging of sensors is helpful for the farmers and others by knowing the place of the yield. The screenshots of the coordinates on MATLAB app and Location on ThingSpeak Channel is shown in Fig.9. 


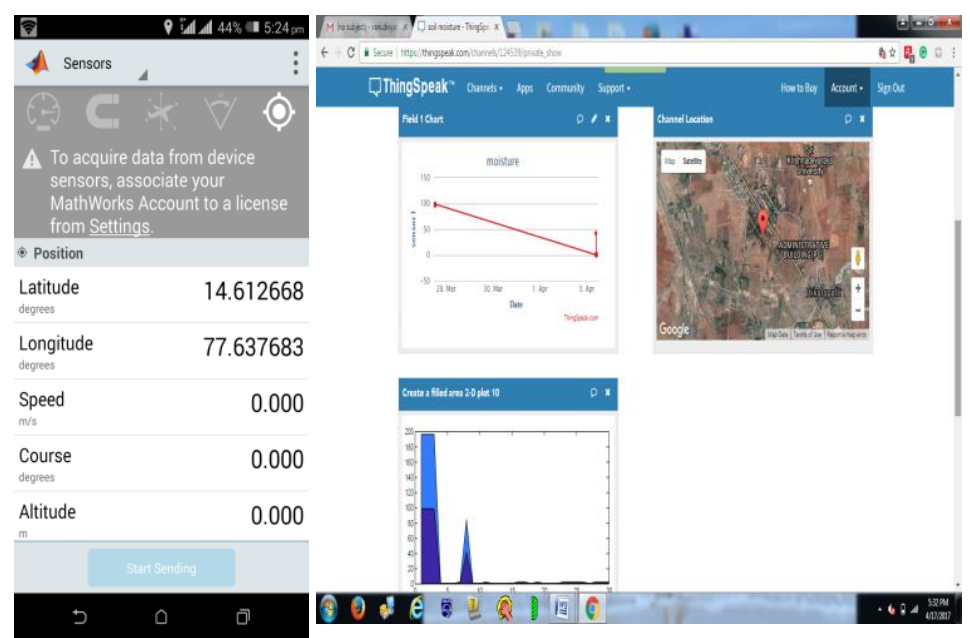

Fig.9. Screenshots of the Coordinates Displayed on MATLAB Mobile Application and the Geotagging of Sensor Data with a Location on Thing Speak Website.

\section{Conclusions}

The low cost, continuous monitoring of soil moisture system was developed. Preliminarily the system was tested on lab environment in different soil conditions of the plant. The system has a capability to continuously measuring the soil moisture values and based on the sensor values the motor is automatically controlled. The moisture values are sent to the ThingSpeak cloud for real-time observation and also send the SMS through GSM module. The graphical representation of sensor values is retrieved and viewed anytime and anywhere in the world.

\section{Acknowledgements}

P. Divya vani is thankful to the Department of Science and Technology (DST), New Delhi, for sanctioning the INSPIRE fellowship and the authors are thankful to DST for sanctioning FIST program in establishing VLSI and Embedded Laboratory in the Department of Physics, Sri Krishnadevaraya University, Anantapuramu.

\section{References}

[1] P. S. Asolkar and U. S. Bhadade, "An Effective Method of Controlling the Greenhouse and Crop Monitoring Using GSM," 2015 International Conference on Computing Communication Control and Automation, Pune, 2015, pp. 214-219. doi: 10.1109/ICCUBEA.2015.47.

[2] L. Li, H. Xiaoguang, C. Ke and H. Ketai, "The applications of WiFi-based Wireless Sensor Network in Internet of Things and Smart Grid," 2011 6th IEEE Conference on Industrial Electronics and $\begin{array}{llll}\text { Applications, } & \text { Beijing, } & \text { 2011, } & \text { 789-793. }\end{array}$ doi: 10.1109/ICIEA.2011.5975693

[3] S. Yin and O. Kaynak, "Big Data for Modern Industry: Challenges and Trends [Point of View]," in Proceedings of the IEEE, vol. 103, no. 2, pp. 143-146, Feb. 2015.doi: 10.1109/JPROC.2015.2388958

[4] S.D.T. Kelly, N.K. Suryadevaraya and S.C. Mukhopadhyay, FIEEE, "Towards the Implementation of 
IoT for Environmental conditions Monitoring in Homes", \{online\} accessed on 03/03/2017. Available:pgembeddedsystems.com/.../IEEE/.../Towards\%20the\%20Implementation\%20of\%20I.

[5] Anthony Faustine, Aloys N.Mvuma, Hector J.Mongi, Maria C.Gabriel, Albino J.Tenge, Samuel B.Kucel, "Wireless Sensor Networks for Water Quality Monitoring and Control within Lake Victoria Basin: Prototype Development", Wireless Sensor Networks, 2014, 6, 281-290.

[6] A. Pramanik, Rishikesh, V. Nagar, S. Dwivedi and B. Choudhury, "GSM based Smart home and digital notice board," 2016 International Conference on Computational Techniques in Information and Communication Technologies (ICCTICT), New Delhi, 2016, pp. 41-46.doi: 10.1109/ICCTICT.2016.7514549

[7] Z. Yuan, Z. Zhang, X. Han, Zhenglu and D. Wang, "Remote Monitor of Farmland Irrigation ThreePhase Motor Based on the GSM Module," 2015 Fifth International Conference on Instrumentation and Measurement, Computer, Communication and Control (IMCCC), Qinhuangdao, 2015, pp. 17791782.doi: 10.1109/IMCCC.2015.378

[8] A. Sathya, B. Arthi, S. Giridharan, M. Karvendan and J. Kishore, "Automatic control of irrigation system in paddy using WSN," 2016 IEEE Technological Innovations in ICT for Agriculture and Rural Development (TIAR), Chennai, 2016, pp. 115-118.doi: 10.1109/TIAR.2016.7801224

[9] Mr. Vinod Kumar P, Dr. U.B. Mahadevaswamy, "Unilateral Vital Signs Monitoring System on IoT", International Journal of Engineering and Manufacturing, 2018, 1, 53-62.

[10] Nureni Asafe Yekini, Uduak Inyang-Udoh, Funmailayo Doherty, “ Open Educational Resources (OER) for Sustainable Development using Autonomic Cloud Computing System", International Journal of Engineering and Manufacturing, 2016, 6,60-68.

[11] CC3200 LaunchPad specifications, [Online].05/02/2015. Available from: http://www.ti.com/general/docs/lit/getliterature.tsp?genericPartNumber=cc3200\&fileType=pdf.

Accessed on 21/04/2015

[12] FC-28 Soil moisture sensor specifications [Online]. Available from: www.nsk electronics.com/soil_moisture_sensor.html. Accessed on 16/01/2016

[13] SIM900A GSM Module \{online\}. Available from: https://elementzonline.com/sim900a-gsm-modemmodule-with-sma-antenna-ttl-output

[14] ThingSpeak \{online\}.Available from: https://thingspeak.com

[15] Srishti Rawal. "IOT based Smart Irrigation System”. International Journal of Computer Applications 159(8):7-11, February 2017.

\section{Authors' Profiles}

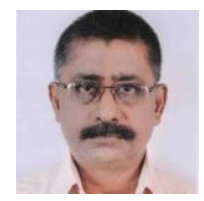

Dr. K.Raghavendra Rao is working as Principal, College of Engineering and Technology and Senior Professor, Department of Physics, S.K.University, Anantapuramu. He has 35 years of Teaching and 32 years of Research experience. He has published 60 Research papers in various National and International journals. Fifteen (16) research students have been awarded Ph.D. degrees and ten students have been awarded M.Phil.Degrees under his guidance. He is a Life member of various societies like ISOI, IETE, and IEEE etc. He completed three Major Research Projects funded by UGC, CSIR, and BRNS. He developed Multichannel Analyzer for Mossbauer Spectroscopy. The same was developed at Indian Institute of Science, Bangalore. He visited Rome, Italy and Bali, Indonesia in connection with Paper presentations at IEEE International Conferences. He has Published 50 Research Papers in National and International Journals and presently writing a text book for a "Practical Training on Embedded System Design", and book Chapters for Elsevier Text book on Big Data. His current interests include Electronic Instrumentation, Embedded System Design and Internet of Things (IoT). 
P.Divya Vani completed M.Sc in Sri Krishnadevaraya University and pursuing Ph.D as a DSTINSPIRE program at Sri Krishnadevaraya University. She published 5 papers which are indexed in Scopus and IEEE journals. Currently, she is working on Internet of Things and Cloud Computing Technology.

How to cite this paper: Palle Divya Vani, Kanchi Raghavendra Rao,"Implementation of Smart Agriculture using CloudIoT and its Geotagging on Android Platform", International Journal of Engineering and Manufacturing(IJEM), Vol.9, No.2, pp.43-53, 2019.DOI: 10.5815/ijem.2019.02.04 